\title{
Firing pattern regulation in hypothalamic vasopressin neurons: roles of synaptic inputs and retrograde signaling
}

\author{
Alexander O Komendantov ${ }^{1 *}$, Ion R Popescu², Jeffrey G Tasker ${ }^{2,3}$ \\ From Nineteenth Annual Computational Neuroscience Meeting: CNS*2010 \\ San Antonio, TX, USA. 24-30 July 2010
}

Magnocellular neurosecretory cells (MNCs) of the hypothalamus release the hormones oxytocin (OT) and vasopressin (VP) into the blood. These cells demonstrate enhancement of hormone release with bursting patterns of electrical activity. OT neurons fire synchronized bursts at long intervals during parturition and milk ejection; VP neurons generate an asynchronous phasic bursting in response to osmotic and cardiovascular stimuli. The mechanisms of bursting activity in VP are not known completely and are believed to be different in vitro and in vivo. Whereas in vitro, phasic bursting in VP neurons appears to be governed by intrinsic deterministic mechanisms, in vivo burst generation and termination significantly depends on synaptic activity. Mounting evidences suggest that retrograde signaling via endocannabinoids (eCBs) plays a prominent role in modulating MNC synaptic activity [1]. Our recent experiments suggest that bursts of action potentials are capable of suppressing glutamatergic input in VP neurons. We also found that blocking eCB receptors increased burst duration and intra-burst action potential frequency, consistent with a potential role in burst termination.

To investigate theoretically the role of synaptic inputs in the phasic bursting activity in VP neurons, we used an updated multicompartmental model of the MNC [2]. The model takes into account MNC morphology and electrotonic properties and includes a set of realistic voltage-gated and $\mathrm{Ca}^{2+}$-activated ion currents, compartmental $\mathrm{Ca}^{2+}$ dynamics and reproduces several of the hallmark characteristics of MNC electrophysiological properties. Phasic bursting in the model is controlled by

* Correspondence: akomenda@gmu.edu

${ }^{1}$ Krasnow Institute for Advanced Study, George Mason University, Fairfax, VA, 22030, USA both intrinsic and synaptic mechanisms: bursts of action potentials arise from the summation of slow depolarizing afterpotentials superimposed on a tonic background activation of glutamatergic synaptic inputs; activitydependent release of a retrograde messenger (eCB) from the dendrites of VP neurons attenuates tonic glutamate release and leads to burst termination. Background synaptic activity was simulated as independent excitatory and inhibitory inputs mediated by AMPA and $\mathrm{GABA}_{\mathrm{A}}$ conductances. Our computational studies also suggest that $\mathrm{GABA}_{\mathrm{A}}$ receptor activation promotes burst firing patterns, and stochastic synaptic inputs play an important role in the modulation of phasic activity in VP neurons.

\section{Acknowledgments}

$\mathrm{NIH}$ grant R01 NS042081.

\section{Author details}

${ }^{1}$ Krasnow Institute for Advanced Study, George Mason University, Fairfax, VA, 22030, USA. ²Department of Cell and Molecular Biology, Tulane University, New Orleans, LA 70118, USA. ${ }^{3}$ Neuroscience Program, Tulane University, New Orleans, LA 70118, USA.

Published: 20 July 2010

\section{References}

1. Di S, Boudaba C, Popescu IR, Weng FJ, Harris C, Marcheselli VL, Bazan NG, Tasker JG: Activity-dependent release and actions of endocannabinoids in the rat hypothalamic supraoptic nucleus. J Physiol 2005, 569:751-760.

2. Komendantov AO, Trayanova NA, Tasker JG: Somato-dendritic mechanisms underlying the electrophysiological properties of hypothalamic magnocellular neuroendocrine cells, A multicompartmental model study. J Comput Neurosci 2007, 23:143-168.

doi:10.1186/1471-2202-11-S1-O1

Cite this article as: Komendantov et al:: Firing pattern regulation in hypothalamic vasopressin neurons: roles of synaptic inputs and retrograde signaling. BMC Neuroscience 2010 11(Suppl 1):O1. 